\title{
A tandem adsorption-catalysis strategy for the removal of copper ions and catalytic reduction of 4-nitrophenol
}

\author{
Muhua Chen, ${ }^{\dagger}$ Yingyun Gao, ${ }^{\dagger}$ Bo Fu, $,{ }^{\dagger}, *$ Fan Yang ${ }^{\ddagger}, *$ \\ $\dagger$ College of Chemical Engineering, Jiangsu Key Lab for the Chemistry \& Utilization \\ of Agricultural and Forest Biomass, Nanjing Forestry University, Nanjing, 210037, \\ China
}

$¥$ School of Management Science and Engineering, Nanjing University of Finance and Economics, Nanjing, Jiangsu, 210023, China

Table S1. The main chemical compositions of purified ATP

\begin{tabular}{cccccccccc}
\hline Composition & $\mathrm{SiO}_{2}$ & $\mathrm{MgO}$ & $\mathrm{Al}_{2} \mathrm{O}_{3}$ & $\mathrm{Fe}_{2} \mathrm{O}_{3}$ & $\mathrm{CaO}$ & $\mathrm{Na}_{2} \mathrm{O}$ & $\mathrm{TiO}_{2}$ & $\mathrm{MnO}$ & $\mathrm{H}_{2} \mathrm{O}$ \\
\hline $\mathrm{wt} \%$ & 53.6 & 11.9 & 7.4 & 2.7 & 2.1 & 0.1 & 0.3 & 0.1 & 10.1 \\
\hline
\end{tabular}

Table S2. The parameters of Langmuir model and Freundlich model

\begin{tabular}{lll}
\hline Type & $\mathrm{K}$ & $\mathrm{R}^{2}$ \\
\hline Langmuir & 3.5208 & 0.9931 \\
Freundlich & 0.7705 & 0.9995 \\
\hline
\end{tabular}

Table S3. Comparison of different catalysts for reduction of 4-NP

\begin{tabular}{llll}
\hline Sample & $k\left(10^{-3} \mathrm{~s}^{-1}\right)$ & $\mathrm{TOF}^{1}\left(\mathrm{~h}^{-1}\right)$ & Ref. \\
\hline $\mathrm{Au} @ \mathrm{CeO}_{2}$ & 13 & 240 & {$[\mathrm{R} 1]$} \\
$\mathrm{Cu} @$ Si nanowires & 11 & 220 & {$[\mathrm{R} 2]$} \\
$\mathrm{Pd} @ \mathrm{GO}$ & 55 & 647 & {$[\mathrm{R} 3]$} \\
$\mathrm{Cu} @$ cellulose & 26 & 125 & {$[\mathrm{R} 4]$} \\
$\mathrm{Cu} @ \mathrm{ATP}$ & 37 & 195 & This work \\
\hline 1 TOF is calculated by the moles of p-nitrophenol reduced per mole of active &
\end{tabular}


[R1] Liu, B.; Yu, S.; Wang, Q.; Hu, W.; Jing, P.; Liu, Y.; Jia, W.; Liu, Y.; Liu, L.; Zhang, J., Hollow mesoporous ceria nanoreactors with enhanced activity and stability for catalytic application. Chem. Commun. 2013, 49 (36), 3757-3759.

[R2] X. Yang, H. Zhong, Y. Zhu, H. Jiang, J. Shen, J. Huang, C. Li, Highly efficient reusable catalyst based on silicon nanowire arrays decorated with copper nanoparticles, J. Mater. Chem. A 2014, 2, 9040-9046.

[R3] Sun, W. N.; Lu, X. F.; Tong, Y.; Zhang, Z.; Lei, J. Y.; Nie, G. D.; Wang, C. Fabrication of Highly Dispersed Palladium/Graphene Oxide Nanocomposites and their Catalytic Properties for Efficient Hydrogenation of Pnitrophenol and Hydrogen Generation. Int. J. Hydrogen Energy 2014, 39, 9080- 9086,

[R4] Song, L.; Shu, L.; Wang, Y.; Zhang, X.-F.; Wang, Z.; Feng, Y.; Yao, J., Metal nanoparticle-embedded bacterial cellulose aerogels via swelling-induced adsorption for nitrophenol reduction. Int. J. Biol. Macromol. 2020, 143, 922-927. 